\title{
The unforeseeable hammerhead ribozyme
} Christian Hammann ${ }^{1}$ and Eric Westhof ${ }^{2 *}$

\author{
Address: ${ }^{1}$ Research Group Molecular Interactions, Department of Genetics, FB 18 Naturwissenschaften, Heinrich-Plett-Str. 40, Universität Kassel, \\ D-34132 Kassel, Germany; ${ }^{2}$ Architecture et Réactivité de l'ARN, Université de Strasbourg, Institut de Biologie Moléculaire et Cellulaire, CNRS, 15 \\ rue René Descartes, F-67084 Strasbourg Cedex, France \\ *Corresponding author: Eric Westhof (e.westhof@ibmc.u-strasbg.fr) \\ FI000 Biology Reports 2009, I:6 (doi: 10.34I0/BI-6)
}

The electronic version of this article is the complete one and can be found at: http://FI000.com/Reports/Biology/content///6

\begin{abstract}
Despite its small size, the complex behavior of the hammerhead ribozyme keeps surprising us, even more than 20 years after its discovery. Here, we summarize recent developments in the field, in particular the discovery of the first split hammerhead ribozyme.
\end{abstract}

\section{Introduction and context}

The hammerhead ribozyme is a small catalytic RNA that is centered on a three-way junction [1]. It was originally discovered in sub-viral plant pathogens, where it is implicated in the processing of multimeric replication circular intermediates [2,3]. The multimeric transcripts self-cleave to generate monomeric linear RNA molecules and subsequently the natural hammerhead ribozymes likely circularize the linear RNAs in the (reverse) ligation reaction. Other genomic locations have also been identified, for example in satellite DNA of amphibians or cave crickets and in distinct loci of the Arabidopsis thaliana genome [4-7]. The biological function of these hammerhead ribozymes, however, has so far remained obscure. For a considerable time, studies were focused on minimal versions of the hammerhead ribozyme, consisting of a catalytic core with a few conserved nucleotides flanked by three helices (Figure 1a), until about five years ago, when it was realized that additional tertiary interacting elements are present in two of the helices (Figure 1b,c). Crucially, these were shown to be essential for the activity of the ribozyme under physiological ionic conditions $[8,9]$. A first crystal structure [10] of such a tertiary stabilized (or 'extended') hammerhead ribozyme showed an intimate network of predominantly non-Watson-Crick base pairs [11] between nucleotides in the (hairpin or internal) loops of stems I and II. These loop-loop interactions are coupled with a subtle rearrangement of the catalytic core compared to earlier structures of minimal ribozymes.

\section{Major recent advances}

While the field concentrated on minimal hammerheads (Figure 1a) with the aim of defining the behavior of the prototypical hammerhead ribozyme, it became clear that no such entity exists. Individual hammerhead ribozymes display a substantial variation in loop or bulge sequences [12] and these sequences sustain the tertiary interactions required for both catalytic activities (cleavage and ligation) under physiological conditions. A recent study systematically investigated the influence of natural loop sequences derived from viroid or satellite RNAs on both the cleavage and ligation reactions [13]. Under identical reaction conditions, a very broad range of catalytic properties were observed, with reaction constants varying by more than $2 \log$ units. This indicates that the peripheral sequence elements (Figure $1 \mathrm{~b}, \mathrm{c}$ ) can exert dramatic effects on the dynamical equilibrium of the local core of the hammerhead ribozyme, the sequence of which is identical in all constructs tested. This observation is not unprecedented as earlier studies had indicated that sequence variations in the core itself could be tolerated, depending on the nature of the hammerhead $[14,15]$. Taken together, these data indicate that the reactions catalyzed by the hammerhead are intrinsically steered by the peripheral loop sequences, allowing the ribozymes to fulfill their biological functions in very different conditions and environments. Thus, the idiosyncrasy of these sequences might allow for regulation of the rolling circle replication of the sub-viral RNAs in which they are located and 
Figure I. Various designs of the hammerhead ribozyme

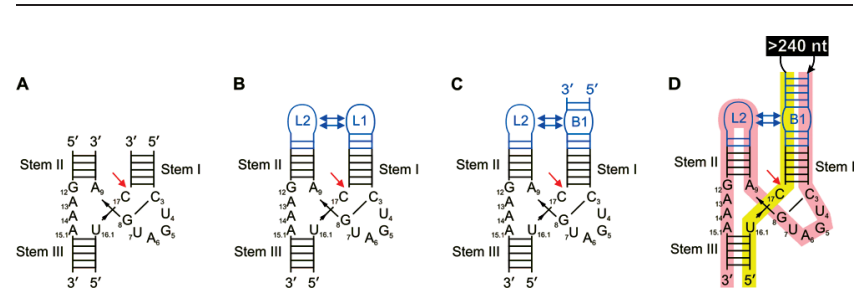

Throughout, the cleavage site is marked with a red arrow and the conventional numbering is used. (a) Minimal version, lacking thetertiary interacting elements as shown for apical loop-loop interactions in (b) and bulge-loop interactions in (c). Recently, a split version of the hammerhead ribozyme was discovered (d) in which the substrate part (yellow) is separated from the ribozyme part (pink) by a long stretch of unrelated nucleotides without impeding the essential tertiary bulge-loop interactions.

influence the ratio of multimers and circular or linear monomers, as recently discussed [13]. The possible roles of the intervening helical stems in the regulation processes are not yet understood.

Despite the strong influence of the peripheral regions on the reaction kinetics of the hammerhead ribozyme, a comparative study shows that the minimal and the tertiary stabilized forms display some clear similarities at the mechanistic level [16]. This very topic, the mechanism of the reaction, for a long time hotly debated, has seen new input from a combined approach using x-ray crystallography and molecular dynamics simulations [17]. This study revealed that a well-ordered $\mathrm{Mn}^{2+}$ ion might induce a solvent-ordering effect promoting the exact positioning of a potentially protonated water or other solvent molecule. This molecule, in turn, might stabilize the transition state by helping to counteract the unfavorable $\mathrm{pK}_{\mathrm{a}}$ of the 2 ' $\mathrm{OH}$ of G8, the hydroxyl group that is considered to be the general acid of the reaction. Closer insight into the transition state has also come from a recent striking crystallographic study [18] that provides structural snapshots of the precatalytic and postcatalytic states of the hammerhead ribozymes derived from the original satellite RNA of the tobacco ringspot virus [3].

Hammerhead ribozymes have been found in other genomes [4-7] as well as those of sub-viral plant pathogens, but the biological roles of such ribozymes are not yet understood. In this regard, an exciting discovery is the split hammerhead ribozyme recently found by means of a database search in the $3^{\prime}$ untranslated region (UTR) of several mammalian mRNAs [19]. In these sequences, initially found in rodent lectin mRNAs, the hammerhead domain consists of a substrate and an enzyme segment, separated by up to hundreds of nucleotides but residing on the same transcript (Figure 1d). Despite their bi-modular design, these new ribozymes feature the tertiary interaction elements that are essential for catalytic activities and probably responsible for the modulation of the activity in individual hammerhead ribozyme sequences as described above.

Because these sequences are located between the stop codon and the polyadenylation signal, a biological function in post-transcriptional gene regulation can be readily envisaged and expression data from fusion constructs support this idea [19]. It is likely that at some level, however, the endogenous split hammerheads are subjected to (allosteric) regulation since otherwise the genes would never be expressed. Modulating the expression levels of the mRNAs that contain the ribozyme might also be achieved by the structural availability of sequences required for cleavage activity, as can be the case in post-transcriptional regulation by the predominantly bacterial riboswitches [20]. Most exciting is the possibility that the enzyme part of the split ribozymes might be active in trans, that is, act on other RNAs that harbor the substrate sequence. Martick et al. [19] point out that several mRNAs that are related to the lectin mRNA contain such a substrate but lack the ribozyme sequence.

\section{Future directions}

The discovery of the split hammerhead ribozymes in the 3' UTR of rodent lectin mRNAs poses the question of whether this is a general and widespread mechanism of post-transcriptional gene regulation. As discussed elsewhere [19], the difficulty of systematically searching for ribozymes in genomes makes it hard to establish such biological regulation. Further genetic and biochemical analyses might reveal whether these sequences indeed might be active naturally as a true enzyme in trans, that is, with the property of multiple turnover. A more general question is that of the parameters that regulate the activity of tertiary stabilized hammerheads, both in the split and the compact forms. For example, can natural changes in the intracellular environment promote or prevent the formation of tertiary interactions in the hammerhead ribozyme, which might be reflected in the accumulation of specific viroid forms? Finding answers to this and related questions will not be trivial and will probably require integrated in vitro and in vivo studies.

\section{Abbreviations}

UTR, untranslated region.

\section{Competing interests}

The authors declare that they have no competing interests. 


\section{Acknowledgments}

$\mathrm{CH}$ is recipient of a DFG Heisenberg stipend and acknowledges support by the DFG (grant HA3459/3-1) and by the Otto-Braun-Fonds.

\section{References}

I. Hammann C, Lilley DM: Folding and activity of the hammerhead ribozyme. Chembiochem 2002, 3:690-700.

2. Hutchins CJ, Rathjen PD, Forster AC, Symons RH: Self-cleavage of plus and minus RNA transcripts of avocado sunblotch viroid. Nucleic Acids Res 1986, 14:3627-40.

3. Prody GA, Bakos JT, Buzayan JM, Schneider IR, Bruening G: Autolytic processing of dimeric plant virus satellite RNA. Science 1986, 23I:1577-80.

4. Przybilski R, Gräf S, Lescoute A, Nellen W, Westhof E, Steger G, Hammann C: Functional hammerhead ribozymes naturally encodedin the genome of Arabidopsis thaliana. Plant Cell 2005, 17:1877-85.

FI000 Factor 3.2 Recommended

Evaluated by Peter Unrau 2 I Jun 2005, Daniel Gallie 17 Aug 2005

5. Ferbeyre G, Smith JM, Cedergren R: Schistosome satellite DNA encodes active hammerhead ribozymes. Mol Cell Biol 1998, I 8:3880-8.

6. Rojas AA, Vazquez-Tello A, Ferbeyre G, Venanzetti F, Bachmann L, Paquin B, Sbordoni V, Cedergren R: Hammerhead-mediated processing of satellite pDo500 family transcripts from Dolichopoda cave crickets. Nucleic Acids Res 2000, 28:4037-43.

7. Zhang Y, Epstein LM: Cloning and characterization of extended hammerheads from a diverse set of caudate amphibians. Gene 1996, 172:183-90.

8. De la Peña M, Gago S, Flores R: Peripheral regions of natural hammerhead ribozymes greatly increase their self-cleavage activity. EMBO J 2003, 22:5561-70.

FI000 Factor 3.0 Recommended

Evaluated by John Burke 7 Apr 2004

9. Khvorova A, Lescoute A, Westhof E, Jayasena SD: Sequence elements outside the hammerhead ribozyme catalytic core enable intracellular activity. Nat Struct Biol 2003, 10:708-12.

FI000 Factor 8.2 Exceptional

Evaluated by Donald Burke 4 Sep 2003, Fritz Eckstein 5 Sep 2003, Barry Stoddard 16 Sep 2003
10. Martick M, Scott WG: Tertiary contacts distant from the active site prime a ribozyme for catalysis. Cell 2006, I 26:309-20.

FI000 Factor 6.5 Must Read

Evaluated by Fritz Eckstein I Aug 2008, Sabine Müller I4 Aug 2006, Eric Westhof 18 Aug 2006

II. Leontis NB, Stombaugh J, Westhof E: The non-Watson-Crick base pairs and their associated isostericity matrices. Nucleic Acids Res 2002, 30:3497-531.

FI000 Factor 3.2 Recommended

Evaluated by Douglas Turner 13 Sep 2002, Evelyn Jabri 9 Oct 2002

12. Westhof $E: A$ tale in molecular recognition: the hammerhead ribozyme. J Mol Recognit 2007, 20:I-3.

13. Shepotinovskaya IV, Uhlenbeck OC: Catalytic diversity of extended hammerhead ribozymes. Biochemistry 2008, 47:703442.

14. Przybilski R, Hammann C: Idiosyncratic cleavage and ligation activity of individual hammerhead ribozymes and core sequence variants thereof. Biol Chem 2007, 388:737-4I.

15. Przybilski R, Hammann C: The tolerance to exchanges of the Watson Crick base pair in the hammerhead ribozyme core is determined by surrounding elements. RNA 2007, 13:1625-30.

16. Nelson JA, Uhlenbeck OC: Hammerhead redux: does the new structure fit the old biochemical data? RNA 2008, I 4:605-15.

FI000 Factor 6 Must Read

Evaluated by Robert Batey 26 Feb 2008

17. Martick M, Lee TS, York DM, Scott WG: Solvent structure and hammerhead ribozyme catalysis. Chem Biol 2008, 15:332-42.

18. Chi YI, Martick M, Lares M, Kim R, Scott WG, Kim SH: Capturing hammerhead ribozyme structures in action by modulating general base catalysis. PLoS Biol 2008, 6:e234.

FI000 Factor 6 Must Read

Evaluated by Pascale Legault 30 Oct 2008

19. Martick M, Horan LH, Noller HF, Scott WG: A discontinuous hammerhead ribozyme embedded in a mammalian messenger RNA. Nature 2008, 454:899-902.

FI000 Factor 10.8 Exceptional

Evaluated by Robert Batey 16 Jul 2008, Thorsten Dieckmann 21 Jul 2008, Sabine Müller 23 Jul 2008, Fritz Eckstein 23 Jul 2008, Andres Jaschke 4 Aug 2008, Barry Stoddard I4 Aug 2008, lan Willis 19 Aug 2008, Eric Westhof 22 Aug 2008

20. Hammann C, Westhof E: Searching genomes for ribozymes and riboswitches. Genome Biol 2007, 8:210 